\title{
Expression Analysis of Flavonoid-related Genes in Green and White Asparagus Spears
}

\author{
Daniel Z. K. Wambrauw ${ }^{1,2}$, Taisuke Kashiwatani ${ }^{2}$, Maiko Matsuhashi², Satomi Yasuhara ${ }^{3}$, Satoshi OKu ${ }^{3}$, \\ Hanako SHIMURA ${ }^{3}$, Kazushige HondA ${ }^{2}$, Tomoo MAEDA ${ }^{2}$ and Takayuki YAMAGUCHI ${ }^{4}$ \\ ${ }^{1}$ The United Graduate School of Agricultural Science, Iwate University, Morioka, Iwate 020-8550, Japan \\ ${ }^{2}$ Faculty of Agriculture and Life Science, Hirosaki University, Hirosaki, Aomori 036-8560, Japan \\ ${ }^{3}$ Graduate School of Agriculture, Hokkaido University, Sapporo, Hokkaido 060-0808, Japan \\ ${ }^{4}$ Iwate Prefecture Government, Morioka, Iwate 020-8570, Japan
}

(Received July 28, 2020; Accepted June 21, 2021)

\begin{abstract}
We performed an expression analysis of flavonoid-related genes such as chalcone synthase $(C H S)$, chalcone isomerase $(C H I)$, flavanone 3-hydroxylase $(F 3 H)$, flavonoid 3'-hydroxylase $\left(F 3^{\prime} H\right)$, and flavonol synthase $(F L S)$ in order to investigate the effects of light exposure on rutin biosynthesis and to determine the rutin content in green and white asparagus spears (Asparagus officinalis). Gene expression levels for the downstream enzymes in the biosynthesis pathway, i.e., $F 3^{\prime} H$ and $F L S$, were significantly higher in green spears than in white spears; however, no significant difference was observed in the expression of upstream genes, such as $C H S, C H I$, and $F 3 H$. Furthermore, the expression of $F L S$ was the lowest among all flavonoid-related genes, which indicates that it might play an important role in rutin regulation. Rutin remained undetected in white asparagus spears, while green asparagus had relatively high amounts present. The results of this study may explain the molecular basis of the relationship between light exposure and rutin biosynthesis in asparagus spears.
\end{abstract}

Keywords : Asparagus officinalis L., rutin, flavonol synthase (FLS) gene, light exposure

\section{INTRODUCTION}

Asparagus (Asparagus officinalis L.) is one of the most widely produced vegetables in the world. Green and white spears originate from the same cultivar but are produced using different cultivation methods in Japan. White spears are shielded from sunlight, i.e., spears that grow in the absence of light, and green and white spears contain different phytochemicals due to these differences (presence vs. absence of light). Green spears contain rutin, while white spears do not have rutin but are rich in saponin (protodioscin) (Maeda et al., 2005, 2008, 2012). Rutin is one of the most significant flavonoids that has been reported to have biological activities, such as hypertension prevention, anti-inflammatory, anti-tumor, anti-bacterial/ viral, and potent radical-scavenging properties. Rutin also has protective effects against capillary fragility and arteriosclerotic vascular changes (Griffith Jr. et al., 1944; Hellerstein et al., 1951; Middleton et al., 2000; Calabro et al., 2005; Guo et al., 2007). Consumer interest in functional foods, such as flavonoids, is increasing; and this interest has been stimulated by the potential health benefits that have arisen from the antioxidant activities of these compounds (Maeda et al., 2006). The main genes involved in rutin biosynthetic pathway are chalcone synthase $(\mathrm{CHS})$, chalcone isomerase $(\mathrm{CHI})$, favanone-3-hydroxylase $(\mathrm{F} 3 \mathrm{H})$, favonoid-3'-hydroxylase $\left(F 3^{\prime} H\right)$, and flavonol synthase
$(F L S)$, glucosyltransferase $(G T)$ and rhamnosyltransferase (RT) (Fig. 1). Studies have revealed that light is one of the most important environmental signals regulating flavonoid biosynthesis (Fuglevand et al., 1996; Jenkins, 1997, 2001; Wade et al., 2001; Maeda et al., 2010; Kopsell and Sams, 2013; Carvalho and Folta, 2014). Supplemental lighting is introduced when asparagus is grown using the "Fusekomi" forcing culture technique, which is a unique cropping technique developed in Japan. With this technique, it was found that the amount of rutin increased as the number of lamps and the duration of light exposure increased, while spear color improved (Wambrauw et al., 2016). Nonetheless, the mechanisms behind this rutin enhancement have not been fully clarified. In addition, only a few studies have addressed the molecular basis of flavonoid metabolism regulation in asparagus (Yi et al., 2019), the effect of light on the accumulation of flavonoid-related genes and the role of light-regulated genes in enhancing rutin production. Therefore, we investigated the effects of light on rutin-related expressions of biosynthetic genes ( $\mathrm{CHS}$, $C H I, F 3 H, F 3^{\prime} H, F L S$ ) and the amount of rutin in green (light-exposed) and white (light-shielded) spears.

\section{MATERIALS AND METHODS}

\section{Plant material}

Two sets of green and white asparagus spear samples were collected for analysis. First, samples cultivated using

Corresponding author : Daniel Z.K. Wambrauw, e-mail : chouzadrak@gmail.com/daniel_zkw@hirosaki-u.ac.jp 


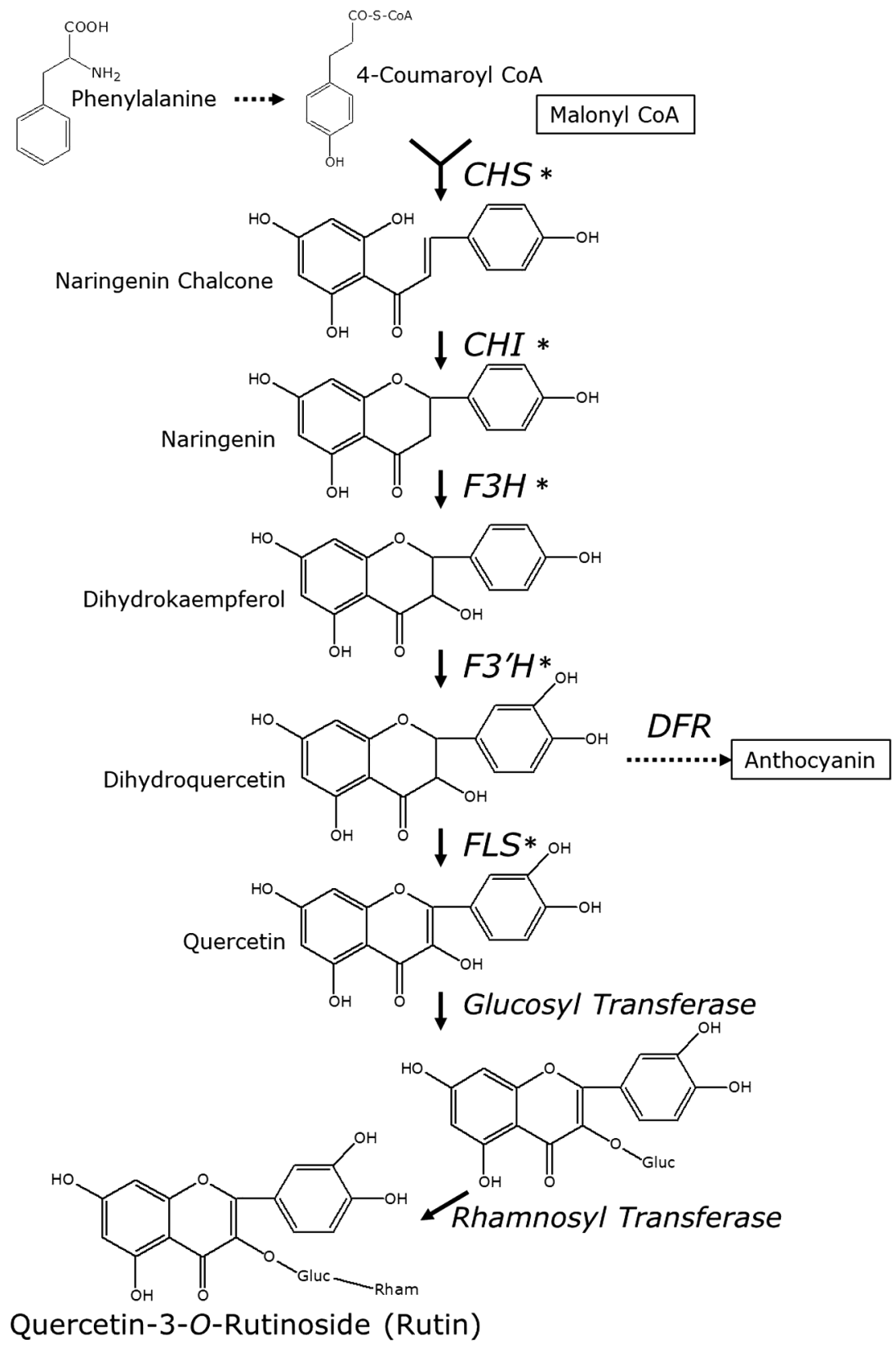

Fig. 1 Schematic of flavonoid biosynthesis in asparagus. Two general classes of the end product are found in asparagus: flavonols (rutin: quercetin-O-rutinoside) and anthocyanins. Enzymes are indicated in bold. CHS: chalcone synthase (CHS catalyzes the first committed step in this pathway), $C H I$ : chalcone isomerase, $F 3 H$ : flavanone synthase, $F 3^{\prime} H$ : flavanone $3^{\prime}$-hydroxylase, $D F R$ : dihydroflavonol 4-reductase, $F L S$ : flavonol synthase, GT: glucosyltransferase, $R T$ : rhamnosyl transferase. Genes marked with * were examined in the present study.

the Fusekomi forcing culture system were collected at Hirosaki University during the winter harvest period. Oneyear-old rootstocks of UC-157 were dug up from an open field at the Iwate Agricultural Research Center on November 19 and planted in heated cultivating beds (length 1.2 $\mathrm{m} \times$ width $0.8 \mathrm{~m} \times$ height $0.45 \mathrm{~m}$ for each plot) set up in a greenhouse in the University field on December 6, 2013. Thirty to forty rootstocks were planted in each plot and covered with rice hull compost. Each test plot planted with green asparagus was covered with a plastic tunnel to maintain warmth. Furthermore, to artificially reproduce the poor light conditions typical of winter, the greenhouse was covered with a shading net to reduce incidental sunlight.
White asparagus is usually produced using the film-cover method (Maeda et al., 2005, 2012); thus, the test plot was covered with a sun-blocking film (Tokan white silver, Tokankosan Co., Japan). The minimum air temperature was kept $>10^{\circ} \mathrm{C}$ using an oil heater, and the soil temperature was kept at approximately $18^{\circ} \mathrm{C}$ using heating wires. The trial was conducted from January 14 to February 24, 2014. During the 6-week harvest period, only samples harvested on February 17 and 24, 2014, were used for subsequent analysis. Only spears grown under natural light without supplemental lighting were used for subsequent analysis. Green and white spears were harvested after reaching $27 \mathrm{~cm}$ in length and were then cut down to $24 \mathrm{~cm}$ 
Table 1 Primer sequences for real-time PCR analysis.

\begin{tabular}{lll}
\hline \multicolumn{1}{c}{ Gene } & \multicolumn{1}{c}{ Forward primer $\left(5^{\prime}-3^{\prime}\right)$} & \multicolumn{1}{c}{ Reverse primer $\left(5^{\prime}-3^{\prime}\right)$} \\
\hline AoActin & ATGGGGGCAGAAGGATGCCCTATG & CCACATCTGTTGGAATGTGCT \\
AoCHS & TGTACCAGCAAGGCTGCTTCG & CTGAATGTGATCCTCACACCG \\
AoCHI & AAGGGGAAGGATGCTGAGGAG & TAGGTTCCGATGGCTTTCCAG \\
AoF3H & CTTCAGGGTGAAGCAGTGCAA & CATGGCCTCGGAGAGAACCAC \\
AoF3dH & GGTGACTTCGTGCCTGCACTGAG & CCTCCTTCAACCTCATCAACACAC \\
AoFLS & CTCACCACCAGGTGAGCTCG & CTGGGGAGCGATCAAACAATG \\
\hline
\end{tabular}

for testing. The second set of asparagus samples were collected from Farm Horo, Shin Hidaka-Cho, Hokkaido, Japan. Green and white spears were harvested during the spring harvest season from 4-year-old UC-157 cultivars. The sample spears were harvested from greenhouses during the latter half of June 2016 and were immediately packed and sent to Hirosaki University for analysis. White spears were produced by covering the greenhouse with a sun-blocking film (Tokan white silver, Tokankosan Co., Japan).

Sample preparation for analysis of gene expression and rutin content

Three to four middle- (M; 13-18 g) to large-sized (L; $18-23 \mathrm{~g})$ spears of green and white asparagus were cut into three equal sections (each section $8 \mathrm{~cm}$ in length). Only the sections with spearheads were evaluated for gene expression analysis and rutin content because rutin content has been reported to be highest in the spearheads (Chin et al., 2002; Maeda et al., 2008). The samples were then vertically cut in half; one half was used for RNA extraction while the other was immediately frozen and then lyophilized to determine rutin content. Total RNA was extracted from about 50-100 $\mathrm{mg}$ of epidermal tissue collected from the samples using an RNeasy Plant Mini Kit (QIAGEN, USA). The cDNA was reverse transcribed from $1 \mu \mathrm{g}$ of the extracted RNA using a QuantiTech Reverse Transcription Kit (QIAGEN, USA). The cDNA samples were stored at $-18^{\circ} \mathrm{C}$ until real-time PCR analysis was performed. Rutin was extracted from $20 \mathrm{mg}$ of the freeze-dried powder obtained from each set of samples using $1 \mathrm{~mL} 80 \%$ methanol, and the extraction was conducted for 3 hours at room temperature. Sample solutions were then centrifuged (10,000 rpm, 10 minutes), and the supernatants were used for further analysis.

\section{Determination of rutin content using HPLC}

Rutin content was determined using high-performance liquid chromatography (HPLC) as described by Maeda et al. (2012). This analysis was conducted using a Waters Sunfire C18 $(4.6 \times 250 \mathrm{~mm})$ column. The mobile phases consisted of $0.1 \%$ trifluoroacetic acid (solvent $\mathrm{A}$ ) and acetonitrile (solvent B). Chromatographic separation was performed by running each sample for 30 minutes at a column temperature of $40^{\circ} \mathrm{C}$, using a linear gradient sys-

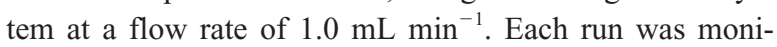
tored at a wavelength of $354 \mathrm{~nm}$ via the UV detectors. The gradient was maintained as follows: 0 minutes, $84 \%$ solvent $\mathrm{A}$ and $16 \%$ solvent $\mathrm{B} ; 20$ minutes, $60 \%$ solvent $\mathrm{A}$ and $40 \%$ solvent $\mathrm{B} ; 30$ minutes, $40 \%$ solvent $\mathrm{A}$ and $60 \%$ sol- vent $\mathrm{B}$; and the post-running time was 10 minutes. Rutin content was calculated using a standard external method with a calibration curve prepared by analyzing the data from rutin standard solutions with concentrations of 0,100 , and 250 ppm (Wako Chemical Co., Japan).

Gene expression analysis

The gene expression levels were evaluated using a real-time polymerase chain reaction (real-time PCR) in a DNA ENGINE OPTICON 2 (BIO-RAD, USA) thermocycler. All target gene primers and reference gene primers (Actin) are presented in Table 1. Real-time PCR was performed using the FastStart Universal SYBR green master mix (Rox) (Roche, USA). The optimized reaction was carried out in a $20-\mu \mathrm{L}$ reaction, containing $10 \mu \mathrm{L}$ of SYBR green master mix (Rox), $0.12 \mu \mathrm{L}$ of each primer (50 pmol), $2 \mu \mathrm{L}(20 \mathrm{ng})$ of the template cDNA, and $7.76 \mu \mathrm{L}$ of sterile water. The PCR protocol was as follows: denaturation for 10 minutes at $95^{\circ} \mathrm{C}$, then 40 cycles of denaturation for 10 seconds at $95^{\circ} \mathrm{C}$, annealing for 30 seconds at $60^{\circ} \mathrm{C}$, and finally one elongation step for 2 minutes at $78^{\circ} \mathrm{C}$. PCR results were calculated as the mean of 3 replicated treatments.

\section{RESULTS AND DISCUSSION}

In the present study, a comparative analysis of flavonoid-related genes and rutin content in green and white spears was performed to investigate how light influences the regulation of rutin. In the first set of samples, the relative gene expression of $\mathrm{CHS}$ and $\mathrm{CHI}$ - the first and second gene in the flavonoid biosynthetic pathway (Fig. 2), followed by $\mathrm{F} 3 \mathrm{H}$ - did not differ significantly between green and white spears (Fig. 3). $F 3^{\prime} H$ expression was significantly higher in green spears than in white spears. The relative gene expression of $F L S$ was also significantly higher in green spears than in white spears (Fig. 3). The $\mathrm{CHS}, \mathrm{CHI}$, and $\mathrm{F} 3 \mathrm{H}$ expression patterns in the second set of samples (harvested from Farm Horo) were found to be similar to the first samples (data not shown). However, the expression of the downstream genes, especially $F L S$, was significantly higher in green spears than in white spears (Fig. 4). The genes that function in the upstream flavonoid biosynthesis pathway were expressed in unpigmented white spears, and their relative gene expression levels were similar to those in green spears. A similar study reported that all flavonoid-related genes were expressed in unpigmented white grapes (Boss et al., 1996). All these genes are precursors for the next genes in the biosynthesis path- 


\section{CHS/ACT}

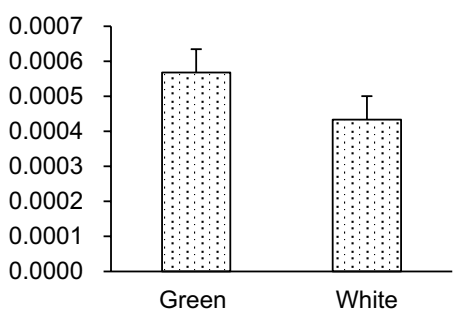

\section{CHI/ACT}

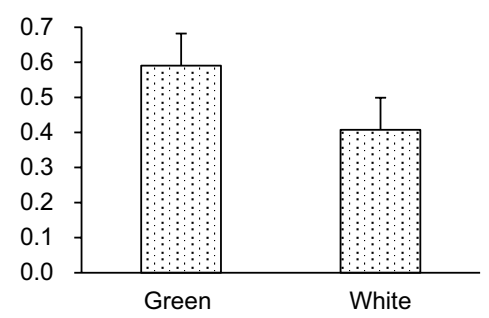

Fig. 2 Expression levels of the earliest genes in the flavonoid biosynthesis pathway. $C H S$ : chalcone synthase, $C H I$ : chalcone isomerase. Green spears were grown under natural light, while white spears were grown under dark conditions. Both green and white spears were harvested during the winter period using the Fusekomi forcing culture system at Hirosaki University. Bars indicate SD. Statistical analysis of the data was performed using $t$-tests $(n=3)$.

F3H/ACT

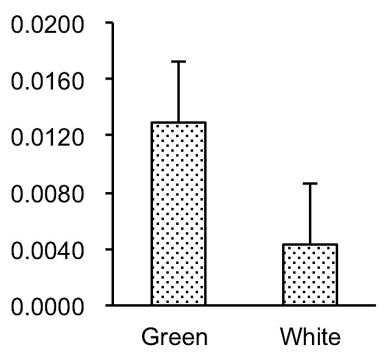

F3'H/ACT

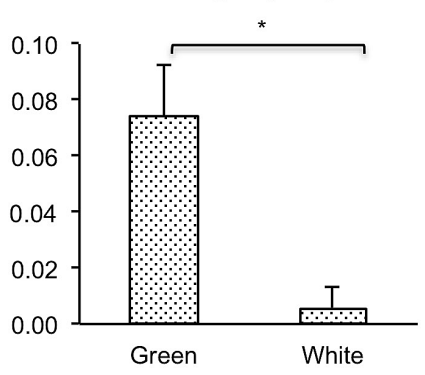

\section{FLS/ACT}

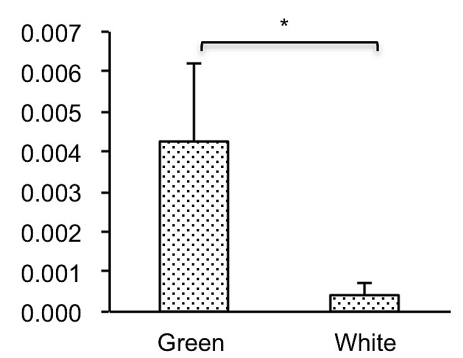

Fig. 3 Expression levels of genes that function in the downstream process of the flavonoid biosynthesis pathway. F3H: flavanone 3-hydroxylase, $F 3^{\prime} H$ : flavonoid $3^{\prime}$-monooxygenase, $F L S$ : flavonol synthase. Green spears were grown under natural light while white spears were grown under dark conditions. Both green and white spears were harvested during the winter period using the Fusekomi forcing culture system at Hirosaki University. Bars indicate SD. Statistical analysis of the data was performed using $t$-tests. ${ }^{*}$ indicate significant differences $(P<0.05, n=3)$.

F3'H/ACT

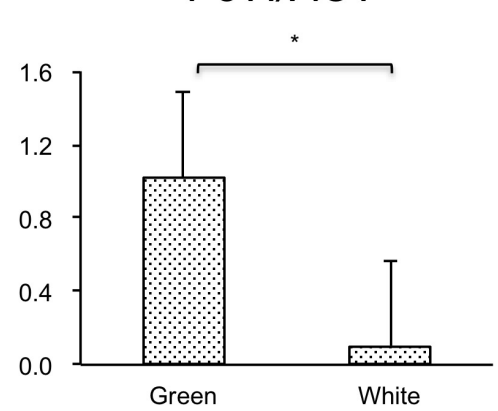

FLS/ACT

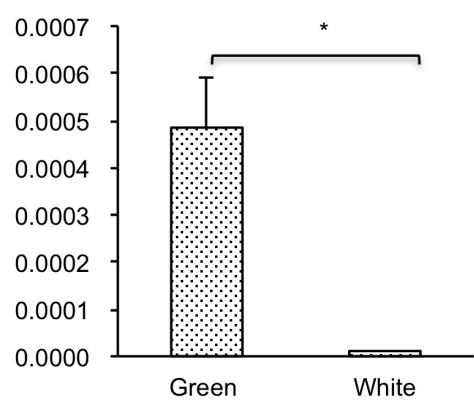

Fig. 4 Expression levels of the last genes in the flavonoid biosynthesis pathway. $F 3^{\prime} H$ : flavonoid $3^{\prime}$-monooxygenase, $F L S$ : flavonol synthase. Green spears were grown under natural light, while white spears were grown under dark conditions. Both green and white spears were harvested during the spring harvest period in Farm Horo, Hokkaido, Japan. Bars indicate SD. Statistical analysis of the data was performed using $t$-tests. ${ }^{*}$ indicate significant differences $(P<0.05, n=3)$. 


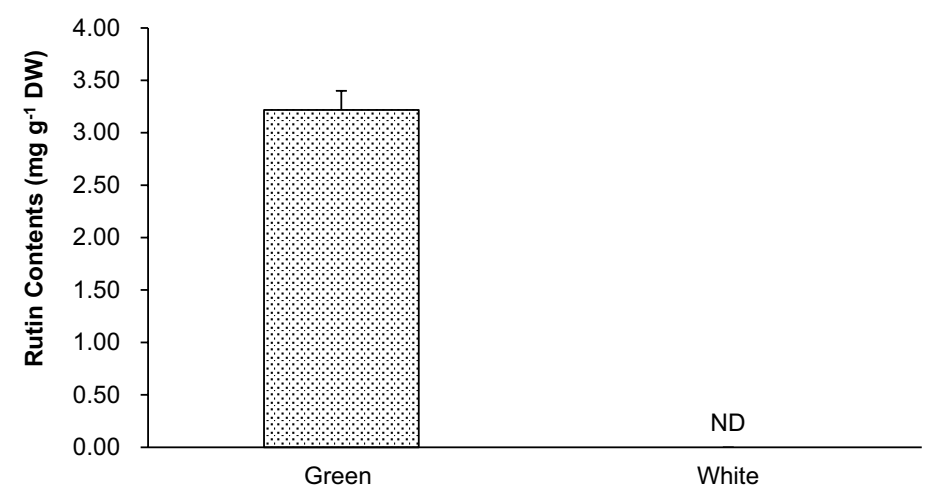

Fig. 5 Rutin content in green and white spears. Green spears were grown under natural light, while white spears were grown under dark conditions. Bars indicate SD $(n=12)$. ND: not detected.

way, which may explain why the expression of these genes occurs in the absence of rutin in unpigmented white spears (Fig. 5). Although all flavonoid-related genes were detected in white spears, the expression levels of $F 3^{\prime} H$ and $F L S$ were significantly lower in white spears than green spears. The expression of FLS in both the first and second sets of samples was lower than that of $F 3^{\prime} H$; it was even quite low in green spears. These results indicated that the expression of $F L S$ was reduced to nearly zero with the absence of light, while FLS expression levels significantly increased in the presence of light (green spears). Since we did not measure the activities of each enzyme, we cannot determine which enzyme is the most important for flavonoid biosynthesis in asparagus spears. However, the results of this study suggest that $F L S$ is an important enzyme that strongly responds to light. $F L S$ is known to be a catalyzer that converts dihydroquercetin to quercetin, which is the starting point of flavonols and also rutin production. Previous studies have also indicated that FLS is the key enzyme in quercetin biosynthesis and is affected by light (Wisman et al., 1998; Pelletier et al., 1999; Li et al., 2010, 2012; Yi et al., 2019). Similarly, a study on lettuce reported that $F L S$ expression was positively correlated with quercetin accumulation upon exposure to light (Ebisawa et al., 2008). These studies support our findings that determined $F L S$ as an important enzyme in asparagus that responds or reacts to the presence or absence of light, and thus regulates rutin biosynthesis.

In this study, we investigated the relationship between light exposure and the expression of flavonoid-related genes in asparagus. Our results indicate that light enhances rutin biosynthesis and that $F L S$ plays an important role in the response of asparagus spears to light.

\section{ACKNOWLEDGMENTS}

This work was supported by JSPS KAKENHI, Grant Number 25450035 .

\section{REFERENCES}

Boss, P. K., Davies, C., Robinson, S. P. $\quad$ 1996. Expression of anthocyanin biosynthesis pathway genes in red and white grapes.
Plant Mol. Biol. 32: 565-569.

Calabro, M. L., Tommasini, S., Donato, P., Stancanelli, R., Raneri, D., Catania, S., Costa, C., Villari, V., Ficarra, P., Ficarra, R. 2005. The rutin/beta-cyclodextrin interactions in fully aqueous solution: spectroscopic studies and biological assays. J. Pharm. Biomed. Anal. 36: 1019-1027.

Carvalho, S. D., Folta, K. M. 2014. Sequential light programs shape kale (Brassica napus) sprout appearance and alter metabolic and nutrient content. Hort. Res. 1: 8 .

Chin, C. K., Garrison, S. A., Ho, C. T., Shao, Y., Wang, M., Simon, J., Huang, M. T. 2002. Functional elements from asparagus for human health. Acta Hortic. 589: 233-241.

Ebisawa, M., Shoji, K., Kato, M., Shimomura, K., Goto, F., Yoshihara, T. 2008. Supplementary ultraviolet radiation B together with blue light at night increased quercetin content and flavonol synthase gene expression in leaf lettuce (Lactuca sativa L.). Environ. Control Biol. 46: 1-11.

Fuglevand, G., Jackson, J. A., Jenkins, G. I. 1996. UV-B, UV$\mathrm{A}$ and blue light signal transduction pathways interact synergistically to regulate chalcone synthase gene expression in Arabidopsis. Plant Cell 8: 2347-2357.

Griffith Jr., J. Q., Couch, J. F., Lindauer, M. A. 1944. Effect of rutin on increased capillary fragility in man. Proc. Soc. Exp. Biol. Med. 55: 228-229.

Guo, R., Wei, P., Liu, W. 2007. Combined antioxidant effects of rutin and vitamin $\mathrm{C}$ in Triton X-100 micelles. J. Pharm. Biomed. Anal. 43: 1580-1586.

Hellerstein, H. K., Orbison, J. L., Rodbard, S., Wilburne, M., Katz, L. N. 1951. The effect of rutin in experimental malignant hypertension. Am. Heart J. 42: 271-283.

Jenkins, G. I. 1997. UV and blue light signal transduction in Arabidopsis. Plant Cell Environ. 20: 773-778.

Jenkins, G. I., Long, J. C., Wade, H. K., Shenton, M. R., Bibikova, T. N. 2001. UV and blue light signalling: pathways regulating chalcone synthase gene expression in Arabidopsis. New Phytol. 151: 121-131.

Kopsell, D. A., Sams, C. E. 2013. Increases in shoot tissue pigments, glucosinolates, and mineral elements in sprouting broccoli after exposure to short-duration blue light from lightemitting diodes. J. Am. Soc. Hort. Sci. 138: 31-37.

Li, X., Park, N. I., Xu, H., Woo, S. H., Park, S. U. $2010 . \quad$ Differential expression of flavonoid biosynthesis genes and accumulation of phenolic compounds in common buckwheat (Fagopyrum esculentum). J. Agric. Food Chem. 58: 1217612181.

Li, X., Thwe, A. A., Park, N. I., Suzuki, T., Kim, S. J., Park, S. U. 2012. Accumulation of phenylpropanoids and correlated gene expression during the development of Tartary buckwheat 
sprouts. J. Agric. Food Chem. 60: 5629-5635.

Maeda, T., Honda, K., Sonoda, K., Motoki, S., Inoue, K., Suzuki, T., Oosawa, K., Suzuki, M. 2010. Light condition influences rutin and polyphenol contents in asparagus spears in the mother-fern culture system during the summer-autumn harvest. J. Jpn. Soc. Hortic. Sci. 79: 161-167.

Maeda, T., Jishi, T., Honda, K., Araki, H., Suzuki, T., Suzuki, M. 2012. Effects of blanching methods on sugar and protodioscin contents of white asparagus spears. J. Jpn. Soc. Hortic. Sci. 81: 166-170.

Maeda, T., Kakuta, H., Ohshima, C., Maekawa, K., Suzuki, T., Oosawa, K. 2006. Additional light supply enhanced polyphenol content and antioxidative capacity of broccoli and red cabbage sprouts grown in an indoor production system. (In Japanese with English abstract) J. SHITA 18: 35-41.

Maeda, T., Kakuta, H., Sonoda, T., Motoki, S., Maekawa, K., Suzuki, T., Oosawa, K. 2008. Differences in antioxidative polyphenols contents of asparagus related to cultivars and seasonal change under various cultural conditions of the motherfern culture. Acta Hortic. 776: 227-234.

Maeda, T., Kakuta, H., Sonoda, T., Motoki, S., Ueno, R., Suzuki, T., Oosawa, K. 2005. Antioxidation capacities of extracts from green, purple, and white asparagus spears related to polyphenol concentration. HortScience 40: 1221-1224.

Middleton, E. J., Kandaswami, C., Theoharides, T. C. 2000. The effects of plant flavonoids on mammalian cells: implica- tions for inflammation, heart disease, and cancer. Pharmacol. Rev. 52: 673-751.

Pelletier, M. K., Burbulis, I. E., Winkel-Shirley, B. 1999. Disruption of specific flavonoid genes enhances the accumulation of flavonoid enzymes and end-products in Arabidopsis seedlings. Plant Mol. Biol. 40: 45-54.

Wade, H. K., Bibikova, T. N., Valentine, W. J., Jenkins, G. I. 2001. Interaction within a network of phytochrome, cryptochrome, and UV-B phototransduction pathways regulate chalcone synthase gene expression in Arabidopsis leaf tissue. Plant J. 25: 675-685.

Wambrauw, D. Z. K., Kashiwatani, T., Komura, A., Hasegawa, H., Narita, K., Oku, S., Yamaguchi, T., Honda, K., Maeda, T. 2016. Effect of supplemental light on the quality of green asparagus spears in winter 'Fusekomi' forcing culture. Environ. Control Biol. 54: 147-152.

Wisman, E., Hartmann, U., Sagasser, M., Baumann, E., Palme, K., Hahlbrock, K., Saedler, H., Weisshaar, B. 1998. Knockout mutants from an En-1 mutagenized Arabidopsis thaliana population generate phenylpropanoid biosynthesis phenotypes. Proc. Natl. Acad. Sci. USA 95: 12432-12437.

Yi, T. G., Yeoung, Y. R., Choi, I. Y., Park, N. I. 2019. Transcriptome analysis of Asparagus officinalis reveals genes involved in the biosynthesis of rutin and protodioscin. PloS One 14: e0219973. 